ORIENTACIONES ESTRATÉGICAS DE LOS INVESTIGADORES EN EL CAMPO DE LA ADMINISTRACIÓN: UN ESTUDIO COMPARATIVO ENTRE REGIONES Y DISCIPLINAS*

\author{
Mario Ernesto Martínez Avella** \\ Iliana Páez Gabriunas****
}

* doi: 10.11144/Javeriana.cao29-52.oeic. Este artículo es producto de la investigación “Factores que influyen en la creación de conocimiento: un estudio sobre la influencia de las orientaciones estratégicas de los investigadores y las redes sociales", financiado por la Universidad de la Sabana desde enero de 2015 hasta diciembre de 2017. Los autores agradecen los aportes conceptuales de Albert Cannella J.R., de W.P. Carey School of Business - ASU y de Eduardo Wills de la Universidad de los Andes. A Juan Daniel Martínez y el equipo que nos colaboró en la elaboración de la BD, y a Isabel Perico por su valioso aporte en la revisión de los textos. El artículo se recibió 28/12/2015 y se aprobó el 30/05/2016. Sugerencia de citación: Martínez A., M. E. y Páez G., I. (2016). Orientaciones estratégicas de los investigadores en el campo de la administración: un estudio comparativo entre regiones y disciplinas. Cuadernos de Administración, 29 (52), 83-114. http:// dx.doi.org/10.11144/Javeriana.cao29-52.oeic

* * Doctor en Administración de la Universidad de los Andes, 2013. Director de la Maestría en Gerencia Estratégica adscrita a la Escuela Internacional de Ciencias Económicas y Administrativas de la Universidad de la Sabana, Bogotá, Colombia.

Correo electrónico: mario.martinez@unisabana.edu.co

*** Doctora en Administración de la Universidad de los Andes, 2013. Directora del Centro de Liderazgo de la Universidad Externado de Colombia, Bogotá, Colombia.

Correo electrónico: iliana.paez@uexternado.edu.co 


\section{Orientaciones estratégicas de los investigadores en el campo de la administración: un estudio comparativo entre regiones y disciplinas}

\section{RESUMEN}

Este artículo presenta un modelo analítico para comprobar las preferencias en los Modos de Producción de Conocimiento de los investigadores en administración. Se aplica el análisis de panel de datos a la producción de 240 investigadores en el periodo 2006-2011 y se encuentra que presentan diferencias significativas en tres orientaciones estratégicas (interdisciplinariedad, aplicación e interacción externa) dependiendo de la disciplina y la región donde trabajan. Los hallazgos muestran que los investigadores en Management se orientan más a la interdisciplinariedad y la interacción externa que los de Finanzas, estos últimos más a la aplicación, y los Latinoamericanos hacen investigación más orientada a la aplicación que los Anglosajones. Se dejan abiertas nuevas perspectivas para estudiar la relevancia de la investigación en administración.

Palabras clave: orientaciones estratégicas, rigor y relevancia de la investigación, investigación en administración, Modo 2.

Clasificación JEL: $\mathrm{O}_{32} \mathrm{O}_{3} 8, \mathrm{M}_{10}$

\section{Strategic orientations of researchers in the field of business and management: $\mathrm{A}$ comparative study between regions and disciplines}

\section{ABSTRACT}

This article presents an analytical model to establish the preferences Business and Management Studies researchers have in Knowledge Production Modes. The panel data methodology is applied on the scientific production of 240 researchers in the time period 2006-2001 and significant differences are found in three strategic orientations (interdisciplinary, applied and external interaction) used by researchers depending on their disciplines and regions of work. The findings show Management researchers are more oriented towards interdisciplinary and external interaction methods than Finance researchers are (the latter are more oriented towards applied methods), and Latin-American researchers tend to do research more oriented towards the applied methods than Anglo-Saxons do. New perspectives are left open to study the relevance of business and management research.

Keywords: strategic orientations, relevance gap, business and management research, Mode 2.

Classification JEL: $\mathrm{O}_{32}, \mathrm{O}_{3} 8, \mathrm{M}_{10}$

\section{Orientações estratégicas dos pesquisadores no campo da administração: um estudo comparativo entre regiões e disciplinas}

\section{ResUMO}

Este artigo apresenta um modelo analítico para comprovar as preferências nos Modos de Produção de Conhecimento dos pesquisadores em administração. Aplica-se a análise de painel de dados à produção de 240 pesquisadores no período 2006-2011 e foi encontrado que apresentam diferenças significativas em três orientações estratégicas (interdisciplinaridade, aplicação e interação externa) dependendo da disciplina e da região em que trabalham. As descobertas mostram que os pesquisadores em Management se orientam mais para a interdisciplinaridade e para a interação externa que os de Finanças, estes últimos mais para a aplicação, e os latino-americanos fazem pesquisa mais orientada à aplicação que os anglo-saxões. Deixam-se abertas novas perspectivas para estudar a relevância da pesquisa em administração.

Palavras-chave: orientações estratégicas, rigor e relevância da pesquisa, pesquisa em administração, Modo 2. 


\section{Introducción}

La reflexión sobre el problema del rigor y la relevancia de la investigación en el Campo de la Administración (CA) no es nueva. Por el contrario, ha hecho parte de los debates cotidianos en las Escuelas de Negocios durante más de cuarenta años y es la fuente de numerosas vertientes de investigación, no solo en las Escuelas de Negocios, sino en los marcos teóricos de la sociología de la ciencia, la ciencia política y los estudios organizacionales en general. Ya en el año 1969, Herber Simon clamaba por una disciplina parecida a la ingeniería o la medicina, para buscar el desarrollo de un campo de estudios más orientado a la aplicación sin debilitar el rigor científico (Simon, 1969). A partir de entonces, la principal preocupación se ha centrado en la búsqueda y la aplicabilidad de la investigación para resolver problemáticas empresariales y sociales concretas, y así contribuir simultáneamente al desarrollo teórico del Campo de la Administración (CA).

Se han presentado muchos constructos y posiciones teóricas con relación a cómo abordar la investigación en el CA, para que sin perder el rigor teórico arroje resultados más claros y aplicables en las organizaciones. En la Academia Norteamericana (Academy of Management) han resultado particularmente influyentes los trabajos de Hambrick (2007) sobre la marcada devoción del Management a la teoría versus su aplicabilidad, la discusión sobre las barreras para el avance de la disciplina adelantada por Pfeffer (1993) y Cannella y Paetzold (1994), el trabajo de Whitley (2000/1984) sobre el estado fragmentado del campo, los trabajos de Rousseau $(2006,2007)$ sobre las recomendaciones para desarrollar el Management con base en evidencia, los trabajos de Huff (2000) y Huff y Huff (2001) sobre los diferentes Modos de Producción de Conocimiento en Management, y el trabajo de Rynes, Gulik y Brown (2007) con su reflexión sobre los mundos separados de académicos y profesionales.

En la Academia Británica el abordaje del tema ha sido aún más prolífico, con trabajos que se han desarrollado a partir de las reflexiones sistemáticas sobre Modos de Producción de Conocimiento (Gibbons, Limoges, Nowotny, Schwartzman, Scotty Trow, 1994), los cuales han nutrido un debate de más de 20 años sobre el problema del rigor y la relevancia de la investigación en el British Journal of Management. Entre otros, se destacan la reflexión de Tranfield y Starkey (1998) en su trabajo seminal sobre política para la investigación en Management, Starkey y Madan (2001) con su defensa a la política de 1998, Van Aken (2005) con su orientación hacia una ciencia de diseño, Pettigrew (1997) quien investiga el dilema del rigor versus la relevancia, Hodgkinson y Starkey (2011) quienes combinan la ciencias de diseño con el realismo crítico y, por su parte, Willmott (2012) con su llamado 
a expandir el marco teórico para entender la relevancia de la investigación. Para el año 2010 ya se habían identificado más de 133 artículos de alto impacto sobre el tema (Nicolai y Seidl, 2010) y aún hoy en día el interés sobre el problema es evidente (Hardeman et al., 2015). En Latinoamérica se identifica el interés sobre el tema desde hace más de quince años (Arocena y Sutz, 2001) y una evidencia actual es la convocatoria en 2016 adelantada por la revista Cuadernos de Administración en Colombia, donde la preocupación central es precisamente el rigor y la relevancia de la investigación en el Campo de la Administración (CA).

La literatura citada en los párrafos anteriores ha resultado casi exclusivamente de la investigación cualitativa y la reflexión de los grandes académicos del CA, quienes han expuesto sus posiciones en diversos congresos y revistas de alto impacto. Sin embargo, dada esta prevalencia en lo cualitativo, las publicaciones en su mayoría adolecen de enfoques analíticos y evidencia empírica que muestre el comportamiento de las preferencias de los investigadores, quienes son los actores fundamentales y determinadores de los modos de producir el conocimiento. Investigaciones recientes argumentan además, que la carencia de investigación empírica cuantitativa obedece a la falta de conceptos operacionalizables sobre los Modos de Producción de Conocimiento, y proponen desarrollar programas de investigación orientados a superar esta deficiencia (Hardeman et al., 2015). Precisamente, nuestro trabajo constituye una contribución en esta dirección.

Nuestra investigación tiene como base el Modelo de las Orientaciones Estratégicas desarrollado por Martínez (2012), donde se hace una primera validación del modelo y se avanza en la reflexión sobre sus consecuencias para la comprensión del problema. En este modelo se sustenta la existencia de tres orientaciones estratégicas de los investigadores en el CA: orientación a la interdisciplinariedad, orientación a la aplicación y orientación a la interacción externa, y la relación de estas orientaciones con la disciplina y la región en la cual trabajan los investigadores. La sustentación de estos constructos resulta importante para conocer las dimensiones individuales que afectan los Modos de Producción de Conocimiento y con estas la comprensión multidimensional del problema de rigor y relevancia en el CA.

En este contexto, nosotros entendemos el CA como el campo que incluye diversas disciplinas relacionadas con la compresión de las organizaciones y que son estudiadas en las principales escuelas de negocios del mundo (Business and Management Studies: BMS según Hodkingson y Starker, 2011). Acogemos además, que el CA se nutre de disciplinas abiertas como comportamiento organizacional, emprendimiento, gerencia estratégica 
y mercadeo, y disciplinas cerradas como la economía y las finanzas, entre otras. Siguiendo la tradición de los estudios cienciométricos y por diversas razones que mostraremos más adelante, nosotros consideramos las disciplinas abiertas como una sola disciplina denominada Management y la disciplina Finanzas como la principal disciplina cerrada que contribuye al CA (Viera y Teixeira 2010; Whitley, 2000/1984). De otra parte, nuestro interés en la incidencia regional sobre las orientaciones estratégicas considera un sentido de región abierto para incluir semejanzas geográficas, sociales e institucionales que definen cierto nivel de homogeneidad académica en el CA (p. ej., la delimitada por los principales países angloparlantes que permiten definir una Región Anglosajona, la correspondiente a la Unión Europea que define una Región Europea y la correspondiente al mundo hispanoparlante que define en gran parte el carácter de la investigación en la Región Latinoamericana).

\section{Antecedentes generales y propósitos de la investigación}

Autores destacados en el British Journal of Management han recomendado hacer la investigación en el campo de la administración (ICA) con orientación a la aplicación, al trabajo interdisciplinario y a la participación de investigadores de diversas organizaciones, con el objeto de superar el llamado "gap de relevancia" o falta de relevancia que tiene la investigación para la práctica de la administración (Tranfield y Starkey, 1998; Starkey y Madan, 2001; Van Aken, 2005; Pettigrew, 1997), buscar mayor impacto de la investigación sobre la efectividad de los negocios (Hodgkinson y Starkey, 2011) y conseguir mayor utilidad social (Willmott, 2012). Estas recomendaciones han tenido como fundamento ideas relacionadas con el llamado Modo 2 de producción de conocimiento, que está enfocado principalmente en la llamada investigación aplicada e interdisciplinaria, y se ha difundido ampliamente entre científicos y formuladores de política para el desarrollo de la ciencia (Gibbons et al., 1994; Hardeman et al., 2015).

La tesis fundamental del Modo 2 ha expuesto que existe una nueva dinámica de la ciencia y la investigación en las sociedades contemporáneas, según la cual está cambiando el modo tradicional de producir el conocimiento llamado Modo 1, cuyo principal objetivo es generar investigación disciplinar. En el Modo 1 la teoría precede la práctica, la investigación se hace en organizaciones científicas y la ciencia se estructura al interior de las disciplinas. En contraste, se considera que los atributos o rasgos básicos del Modo 2 están constituidos por: producción de conocimiento en el contexto de aplicación, transdisciplinaridad, heterogeneidad y diversidad de lugares en la producción de conocimiento, 
responsabilidad y reflexividad social, y un control de calidad amplio más allá del sistema de pares académicos (Hessels y van Lente, 2008).

La recomendación de hacer la investigación con los atributos del Modo 2 está ampliamente generalizada incluso más allá del CA. Campos relacionados con las ciencias ambientales, la sociología y la política pública de la ciencia son algunos ejemplos de la promoción que han tenido los atributos del Modo 2 (Hodgkinson y Starkey, 2011; Albert, 2003), pues se ha considerado que son estos los que mejor contribuyen a mejorar problemáticas sociales y empresariales en el mundo contemporáneo. Igualmente, el impacto del Modo 2 en las políticas de ciencia y tecnología en el ámbito mundial ha tenido un gran eco y, de manera particular, en los países latinoamericanos. En Colombia, por ejemplo, la formulación de metas para el desarrollo se ha visto acompañada de un fuerte discurso que promueve la investigación orientada a la aplicación y se considera el Modo 2 como una de las exigencias para hacer investigación que permita responder a los problemas de la sociedad (Conpes 3582, 2009; Malaver, 2006). En términos generales, aunque el discurso sobre el Modo 2 ha sido complementado por las diversas teorías sobre los Sistemas de Innovación (Triple Hélice, Sistemas Nacionales de Innovación, Ciencia Posnormal y Capitalismo Académico, entre otros), se sigue considerando un enfoque clave para entender y superar la brecha entre la teoría y la práctica en administración (Bartunek, 2011; Hodgkinson y Starkey, 2011; Pascal, Thomas y Romme, 2013).

No obstante, la literatura sobre la ICA muestra también que el Modo 2 no es una tendencia homogénea de los sistemas de investigación, y la combinación de los diferentes atributos asociados con el Modo 1 y el Modo 2 puede dar origen a diferentes Modos de Producción de Conocimiento (Burgoyne y Turnbull, 2006; Das, 2003; MacLean, MacIntosh y Grant, 2002; Huff, 2000; Huff y Huff, 2001; Hardeman et al., 2015). Al comparar los Sistemas de innovación, el trabajo de Hardeman refuta la prevalencia general de los atributos del Modo 2 como principio de todos los sistemas de innovación contemporáneos, ya que el Modo 1 aún prevalece. Muestra además, que hay diferencias en el desarrollo de los rasgos del Modo 2 en función de las distancias cognitivas, institucionales, geográficas y sociales de los diferentes actores que intervienen en los proceso de producción de conocimiento (Hardeman et al., 2015).

De otra parte, se ha observado que tres de los cinco atributos básicos considerados en el Modo 2: la producción de conocimiento en el contexto de aplicación, la transdisciplinaridad, y la heterogeneidad y diversidad de lugares en la producción de conocimiento, constituyen variables de comportamiento individual que definen elecciones o prefe- 
rencias que tienen los investigadores (Martínez, 2012). Estas inciden directamente en la forma de producción de conocimiento y la percepción que se tiene del problema del rigor y la relevancia. Estos tres atributos han permitido conceptualizar tres orientaciones estratégicas de los investigadores que se desarrollan con el objeto de buscar recursos y posiciones competitivas en el mercado del trabajo científico, tales como la incorporación de diversos conocimientos, la búsqueda de relevancia en el campo científico y la adquisición de recursos económicos escasos. Dichas orientaciones estratégicas son la orientación a la interdisciplinariedad, la orientación a la aplicación y la orientación a la interacción externa.

A continuación exponemos las definiciones de partida de estas orientaciones. (i) la orientación a la interdisciplinariedad es un requisito para la transdisciplinariedad de los sistemas de investigación y está determinada por la diversidad de disciplinas que un investigador incorpora en sus productos de investigación; (ii) la orientación a la aplicación es la mayor o menor tendencia que tiene un investigador a producir conocimientos que expliquen las prácticas de la organización social y contribuyan al cambio de las actividades comerciales, industriales, del gobierno o la sociedad en general; y (iii) la orientación a la interacción externa es la tendencia de los investigadores a buscar coautores provenientes de lugares diferentes a su sitio de trabajo habitual, tales como diferentes departamentos académicos, otras universidades u organizaciones; esto propicia la creación de redes de investigación y enriquece el marco teórico y práctico de la misma.

Con base en estos constructos y la mencionada literatura sobre la producción de conocimiento y la ICA, conjeturamos que las orientaciones estratégicas que tienen los investigadores dependen de las características de las disciplinas en las cuales hacen investigación y las características geográficas, sociales e institucionales relacionadas con su lugar de trabajo, lo cual incide en que se privilegien unos atributos de los modos de producción de conocimiento sobre otros. Argumentamos así, que los investigadores en el CA no siguen una tendencia conjunta hacia todos los atributos del Modo 2, sino que se orientan, en mayor o menor medida, hacia los diferentes atributos de los Modos de Producción de Conocimiento dependiendo de factores individuales. Esta condición, que de alguna manera ya ha sido reconocida en la literatura, favorece el abordaje equilibrado del problema del rigor y la relevancia, evitando los extremos que han sobredimensionado o rechazado el pragmatismo del Modo 2 u olvidado en algunos casos la necesidad de acoger rasgos del Modo 1 en algunas disciplinas para mantener el rigor y buscar su desarrollo (Huff y Huff, 2001). 
En síntesis, sustentamos la existencia de tres orientaciones estratégicas de los investigadores en el campo de la administración, y explicamos la relación de dichas orientaciones con dos variables: la disciplina de investigación, según sea Management o Finanzas, y la región donde trabajan predominantemente los investigadores (anglosajona, europea o latinoamericana). Con esto, esperamos aportar nuevos elementos analíticos que faciliten la compresión de los Modos de Producción de Conocimiento en Administración, y dar apertura a una nueva perspectiva sobre las variables de nivel individual que afectan la comprensión del problema del rigor y la relevancia de la Investigación en Administración.

\section{Marco teórico}

Dos antecedentes fundamentales de esta investigación son el Modelo de posicionamiento estratégico de los investigadores que hace énfasis en la autonomía y el carácter emprendedor del investigador (Kurek, Geurts y Roosendaal, 2007; Zalewska-Kurek, 2008), y el Modelo de las orientaciones estratégicas que se centra en las preferencias de los investigadores para buscar recursos, legitimidad y productividad (Martínez, 2012). En el primer caso se desarrolló un modelo para analizar las posiciones estratégicas de los investigadores en su ambiente y evaluar su impacto en la creación y diseminación del conocimiento. En esos trabajos se adelantaron estudios de tipo trasversal con datos obtenidos a partir de encuestas y se llegó a la categorización sobre un plano cartesiano de cuatro posiciones estratégicas de los investigadores sobre dos dimensiones: interdependencia estratégica y autonomía organizacional (Modo 0, Modo 1, Modo 2 y Modo 3). El Modelo de las orientaciones estratégicas es homólogo al Modelo de posicionamiento estratégico pero se ha desarrollado sobre tres dimensiones (orientación a la interdisciplinariedad, orientación a la aplicación y orientación a la interacción externa, ver figura 1). Martínez (2012) sustentó el paralelo entre la interdependencia estratégica y la orientación a la interacción externa y la correlación negativa entre la autonomía organizacional y la orientación a la interdisciplinariedad, con lo cual se aprecia el carácter homólogo de los modelos en dos dimensiones. Adicionalmente el Modelo de las orientaciones estratégicas reconoce la orientación a la aplicación como una tercera dimensión que se deriva de la interacción entre el uso de la investigación y la interdependencia estratégica considerada por Kurek et al. (2007).

El Modelo de posicionamiento estratégico no contempla de manera explícita la orientación a la aplicación, pero considera que la relevancia práctica y la preferencia por el uso de la investigación son aspectos de la interdependencia estratégica, pues están implícitos en la elección que hacen los investigadores cuando deciden compartir sus productos cien- 
tíficos. La reflexión sobre el modelo de Kurek et al. (2007) llevó a Martínez (2012) a la construcción de un paralelo entre los dos modelos, con base en el significado que tienen las puntuaciones de los investigadores en las dimensiones sustentadas por Kurek et al. (2007) y las orientaciones estratégicas y su relación con los Modos de Producción de Conocimiento en los cuales pueden posicionarse los investigadores. Los dos modelos han mostrado la existencia de unas posiciones de los investigadores que, en mayor o menor medida, están orientadas a las demandas del ambiente y las relaciones externas, y otras posiciones que están orientadas al desarrollo de las capacidades internas del campo de estudios y la creación de la demanda por la investigación.

El modelo de Kurek et al. (2007) y el modelo de Martínez (2012) confluyen en una consideración común: los investigadores académicos tienden a desarrollar preferencias por determinados rasgos de los modos de hacer investigación para la creación de conocimiento, buscando ya sea la posición en las dimensiones interdependencia estratégica y autonomía organizacional (en el caso del modelo de posicionamiento estratégico) o la mayor o menor orientación a la interdisciplinariedad, la aplicación y la interacción externa (en el caso del modelo de las orientaciones estratégicas). En este artículo utilizaremos el Modelo de las Orientaciones Estratégicas desarrollado por Martínez (2012), por considerarlo más incluyente y con mayor aplicabilidad en la reflexión sobre el problema de rigor y relevancia de la investigación en el Campo de la Administración (CA).

\begin{tabular}{|l|l|}
\hline $\begin{array}{l}\text { Antecedentes } \\
\text { Disciplina de investigación } \\
\text { Minangement }\end{array}$ & $\begin{array}{l}\text { Orientaciones estratégicas de los } \\
\text { investigadores }\end{array}$ \\
Región de trabajo & $\begin{array}{l}\text { Orientación a la } \\
\text { interdisciplinariedad }\end{array}$ \\
$\begin{array}{ll}\text { Latinoamérica } \\
\text { Anglosajona } \\
\text { Europea }\end{array}$ & $\begin{array}{l}\text { Orientación a la aplicación } \\
\text { Orientación a la interacción } \\
\text { externa }\end{array}$ \\
\hline
\end{tabular}

Figura 1. Modelo de las orientaciones estratégicas de los investigadores

Fuente: elaboración propia.

Teniendo en cuenta estos antecedentes, sostenemos que la producción de conocimiento se realiza con diversas combinaciones de los atributos de los modos de producción, no solo por las exigencias del ambiente, sino también porque los investigadores se 
orientan a dichos atributos como resultado de un conjunto de condiciones personales y de sus campos de investigación (Martínez y Wills, 2010; Hambrick, 1994). Esto hace que se privilegien unos rasgos de los modos de producción sobre otros, lo cual nos permite formular un conjunto de hipótesis que presentamos a continuación.

\subsection{Hipótesis}

En primer lugar, buscamos comprobar la presencia de las Orientaciones Estratégicas de los Investigadores en el Campo de la Administración como una hipótesis fundamental aún no difundida en la literatura y sustentar las relaciones propuestas en nuestro modelo. En consecuencia, sostenemos que un primer antecedente de las orientaciones estratégicas de los investigadores en el CA es la disciplina en la cual estos hacen investigación. Para demostrarlo hemos seleccionado las disciplinas que ofrecen la mayor posibilidad de contraste desde el punto de vista cognitivo, institucional y organizacional: Management y Finanzas. De otra parte, consideramos como un segundo antecedente la región donde han trabajado predominantemente los investigadores. Los países incluidos en cada región son: Estados unidos, Canadá e Inglaterra (región anglosajona); Francia, Alemania, Holanda, Italia, España, Suiza, Suecia y Dinamarca (Europa) y Colombia, México, Chile, Argentina, Brasil, Ecuador, Perú, Venezuela y Costa Rica (Latinoamérica).

\subsubsection{LAS DISCIPLINAS DE INVESTIGACIÓN Y LAS ORIENTACIONES ESTRATÉGICAS DE LOS INVESTIGADORES}

Los trabajos más representativos sobre los Modos de Producción de Conocimiento en el CA se han dirigido en gran medida a formular políticas para el desarrollo de la investigación recomendando hacer la investigación con los atributos de Modo 2 (Tranfield y Starkey, 1998; Starkey y Madan, 2001; Van Aken, 2005; MacLean, MacIntosh y Grant, 2002; Pascal et al., 2013), debido a la necesidad de buscar mayor aplicación de la investigación. No obstante, otras perspectivas han considerado la importancia de reconocer y considerar la combinación de los atributos del Modo 1 y el Modo 2 para orientar la investigación en las escuelas de negocios (Withley, 2000/1984; Huff, 2000; Huff y Huff, 2001; Kurek et al., 2007; Martínez, 2012, etc.), teniendo en cuenta que el interés teórico es muy importante en algunas disciplinas que contribuyen al CA ( $p$. ej., la teoría organizacional).

En general, considerar que todas las ciencias tienden a los atributos del Modo 2 es una posición refutada desde el punto de vista empírico, como lo muestran Metzger y Zare 
(1999), Llerena y Meyer-Krahmer (2003), Van Leeuwen y Tijssen (2000) y Bonaccorsi (2008). Otras evidencias se encuentran en Jansen, von Götz y Heildler (2008, 2010a, 2010 b) quienes mostraron que hay diferencia en la estructura de las redes de colaboración entre los campos de conocimiento (p. ej., nanociencia, astronomía y economía) y consideraron que los campos del conocimiento tienen un desarrollo mixto de los atributos del Modo 1 y el Modo 2, con lo cual se favorece su desarrollo disciplinar. También Qin, Lancaster y Allen (1997) mostraron que hay diferentes niveles de interdisciplinariedad según la disciplina, en tanto Albert (2003) mostró evidencias de la presencia mixta del Modo 1 y el Modo 2 comparando los campos de la sociología, la economía y la ingeniería. Estos últimos encontraron que hay prevalencia de los rasgos del Modo 1 en la Economía y la Ingeniería, y prevalencia de los rasgos del Modo 2 en la Sociología, por lo cual refutaron la existencia de modos puros en alguna disciplina.

En el CA, Viera y Teixeira (2010) estudiaron las disciplinas de Finanzas y Management y encontraron diferencias significativas en su forma de producir el conocimiento. Ellos encontraron que Finanzas es una disciplina relativamente más organizada y establecida que la disciplina del Management, y que esta última es una disciplina más hibrida en la composición del conocimiento que utiliza y que produce. Los resultados del trabajo de Vieira y Teixeira concuerdan con las afirmaciones de Whitley (2000/1984), quien considera que Finanzas es una disciplina más formalizada, con técnicas y enfoques altamente restringidos, a diferencia del Management que se ha desarrollado sobre un conjunto más diverso de metas teóricas y habilidades de investigación. Whitley explicó estas diferencias con base en dos dimensiones prácticas de los investigadores: la dependencia mutua entre los coautores y la incertidumbre de la tarea. Sus reflexiones permiten concluir que el Management tiene un alto grado de incertidumbre en la tarea y bajo grado de dependencia de los colegas, mientras Finanzas tiene un alto grado de dependencia de los colegas y un bajo grado de incertidumbre de la tarea (Whitley 2000/1984; Vieira y Teixeira, 2010). En consecuencia, los investigadores en Finanzas, a diferencia de los investigadores en Management, fundamentan su prestigio en el uso riguroso de métodos y técnicas cuantitativas dominadas por comunidades más reducidas y, en gran parte, desarrolladas al interior de su propia disciplina. Esto los lleva a desarrollar lazos más fuertes entre los colegas, y a moverse sobre temas y agendas de investigación más definidas.

Estas reflexiones permiten deducir que la disciplina de Management, en comparación con la disciplina de Finanzas, está más orientada a crear conocimiento interdisciplinario. Llevando esta consideración al nivel individual, planteamos la primera hipótesis. 
Hipótesis 1. Los investigadores en las disciplinas de Finanzas y Management presentan diferencias significativas en la orientación a la interdisciplinariedad. De tal forma que los investigadores en Management estarán más orientados a la interdisciplinariedad que los investigadores en Finanzas.

De otra parte, como bien se reconoce en la literatura, hay una "marcada devoción de la disciplina de Management por la teoría, en comparación con otras disciplinas que contribuyen al campo de la administración" (Hambrick, 2007, p. 1346). Esto es particularmente válido cuando se compara Management con Finanzas. Si bien es cierto hay una preocupación permanente en Management por la solución de problemas en las organizaciones, la relación de las Finanzas con los problemas prácticos es más directa, hasta el punto que el concepto de ingeniería financiera (como ciencia aplicada) se ha vuelto sinónimo de las soluciones óptimas a los problemas económicos y financieros (Finnerty, 1988). En consecuencia, podemos considerar en el nivel individual una segunda hipótesis.

Hipótesis 2. Los investigadores en las disciplinas de Finanzas y Management presentan diferencias significativas en la orientación a la aplicación. De tal forma que los investigadores en Finanzas estarán más orientados a la aplicación que los investigadores en Management.

Un tercer rasgo individual asociado a la disciplina objeto de investigación es la preferencia por hacer coautoría con colegas localizados del mismo departamento donde trabaja el investigador (colaboración intra-organizacional), o la orientación a publicar con colegas que trabajan en otros departamentos e incluso en diferentes organizaciones (McFadyen y Cannella, 2005). Nosotros homologamos este rasgo con la orientación a la interacción externa y encontramos que la colaboración intra-organizacional se favorece cuando hay proximidad institucional. Es decir, cuando los investigadores comparten el mismo conjunto de valores y normas (Hardeman et al., 2015). Esta consideración sumada a la preferencia de los investigadores en Finanzas por coautores de su misma disciplina, nos llevan a proponer una tercera hipótesis.

Hipótesis 3. Los investigadores en las disciplinas de Finanzas y Management presentan diferencias significativas en la orientación a la interacción externa. De tal forma que los investigadores en Management estarán más orientados a la interacción externa que los investigadores en Finanzas. 


\subsubsection{LA REGIÓN DE TRABAJO Y LA ORIENTACIÓN A LA APLICACIÓN}

Como ya lo hemos planteado, se puede reconocer que otro antecedente que influye en las orientaciones estratégicas de los investigadores es la región donde ellos trabajan en forma predominante. Desde la perspectiva académica, puede inferirse, por ejemplo, que los investigadores latinoamericanos tienen una mayor orientación a la aplicación que los investigadores anglosajones, dada la poca atención que le han puesto los primeros al desarrollo conceptual del CA (Calderón et al., 2010) y la alta preocupación que han demostrado los segundos por el desarrollo teórico (Hambrick, 2007). Esta consideración tiene correlación con la política para la ciencia y la tecnología que predomina en los países latinoamericanos, pues estos consideran fundamental el fortalecimiento de la investigación aplicada a la solución de problemas en las empresas y su desarrollo tecnológico (Monroy, 2006, p. 158) y dan prioridad al desarrollo de proyectos de investigación aplicada que busquen mejorar la competitividad (Conpes 3582, 2009, p. 164).

De otra parte, los resultados específicos de estudios para algunos países de la región como México y Chile y sus discursos sobre política para la ciencia y la tecnología también muestran la tendencia general de la producción científica latinoamericana hacia un mayor desarrollo de la investigación orientada a la aplicación (Contreras, Edwards y Mizala, 2006). El trabajo de Monroy (2006) sobre el Sistema de Ciencia Tecnología e Innovación Colombiano encontró que las prioridades en acciones y actividades dadas por los grupos de investigación tienen una proporción de 3 a 1 entre la investigación aplicada y la investigación básica (Monroy, 2006). Un trabajo más reciente de Naranjo y Calderón (2010) sobre la investigación en innovación en Colombia y México mostró que el 54\% de los artículos publicados presentaban resultados basados en datos empíricos, mientras solo el $46 \%$ eran de carácter teórico y, de estos el $46 \%$ fueron desarrollados en el campo de la administración. Nosotros presumimos que la mayoría de estos últimos presentan baja preocupación por el desarrollo teórico del CA.

En general, la comunidad académica latinoamericana (y en particular la colombiana) ha reconocido el bajo desarrollo de la investigación teórica en comparación con la investigación mundial, y el poco desarrollo de una comunidad científica que se interese en los aspectos conceptuales del CA (Calderón et al., 2010, p. 92). En el estudio adelantado por Calderón y sus colegas se encontró que la comunidad de investigadores en administración reclama "mayor cantidad y calidad en los trabajos teóricos que tienda a incrementar la calidad conceptual de la disciplina" (ibíd., p. 109). Teniendo en cuenta el fuerte énfasis que tienen las políticas gubernamentales hacia la investigación aplicada en Latinoamé- 
rica y, a la vez, el incipiente estado de desarrollo del CA en esta región (Malaver, 2006; Contreras et al., 2006) es presumible que el nivel de producción teórica sea más bajo en Latinoamérica que en otras regiones del mundo. Sobre estas reflexiones es de esperarse que los investigadores latinoamericanos se encuentren más orientados a la aplicación que los investigadores de otras regiones (anglosajones y europeos). Así, formulamos la cuarta hipótesis.

Hipótesis 4. Los investigadores latinoamericanos en el campo de la administración están más orientados a la aplicación que los investigadores anglosajones y europeos.

El conjunto de hipótesis y relaciones propuestas en este artículo se resumen en la figura 2.

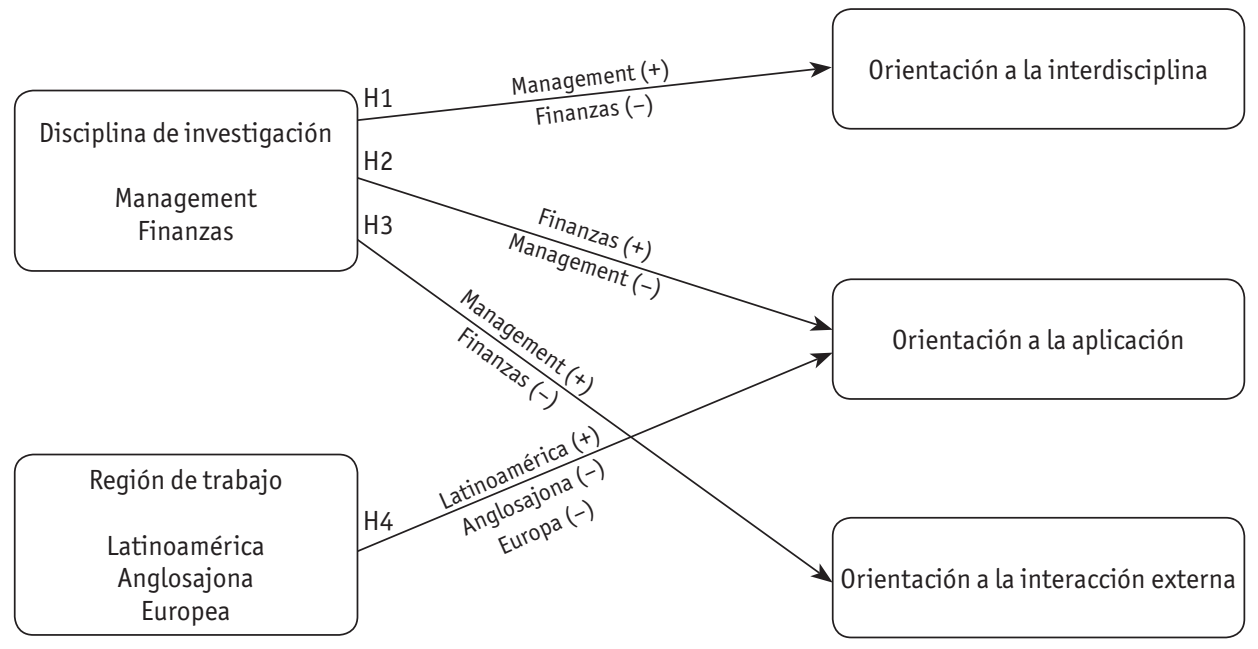

\section{Figura 2. Hipótesis}

Fuente: elaboración propia.

\section{Métodos}

Los métodos utilizados tienen como base la aplicación de la técnica de Panel de Datos sobre el periodo 2006-2011. Para la selección de los datos desarrollamos un método de minería y recolección de información que nos permitió seleccionar la muestra a partir de la base de datos ISI WoS y datos del Journal Citation Report. Con base en estos datos calculamos las diferentes medidas de las variables y aplicamos técnicas estadísticas utilizando el paquete STATA 11.0 para probar las hipótesis propuestas. 
Los datos del panel contienen las orientaciones estratégicas de los investigadores, las variables demográficas consideradas en las hipótesis y dos variables de control: el nivel de experiencia de los investigadores y la posesión del título de doctor. El tipo de investigación, en consecuencia, implica la prueba de las hipótesis teniendo en cuenta el comportamiento en el tiempo de las variables y las variaciones entre los individuos para cada una de ellas (cross-sectional time series).

\subsection{Características de la población y la muestra}

La población objeto de estudio son los investigadores que tienen publicaciones registradas en la Base de Datos ISI en las disciplinas seleccionadas (Management y Finanzas) y el horizonte de tiempo considerado (2006-2011). Sobre esta población, se seleccionó una muestra aleatoria de 250 investigadores con proporciones de $50 \%$ para cada disciplina, segmentada según la región donde trabajan los investigadores y con un número de investigadores proporcional a los aportes que hizo la región a las publicaciones ISI en el periodo estudiado. Dado el escaso número de investigadores latinoamericanos con publicaciones registradas en ISI en el periodo de estudio y su bajo nivel de producción (uno o dos artículos), fue necesario acotar la población seleccionando los investigadores posicionados en el cuartil superior con mayor registro de artículos en cada región, para poder hacer comparables las regiones y mantener la potencia de la muestra. A partir de la población acotada se seleccionaron sub-muestras con valores entre el $25 \%$ y el 35\% del total de la población acotada y, de esta forma, se conformó una muestra general segmentada y estructurada con una potencia aproximada de $85 \%$ para un $\alpha=0,1$ (error tipo I); siguiendo los criterios de Cohen (1997) para un tamaño del efecto entre los grupos principales de 0,35 . Ver la tabla 1.

Una vez depurada la muestra para descartar los autores con resultados fuera de contexto - outlayers (ej., medidas periféricas no comparables o más de dos periodos sin producción de conocimiento) y teniendo en cuenta la estructura definida, la muestra total quedó limitada a 240 autores (96\% de la muestra inicial) pertenecientes a las tres regiones objeto de estudio. Teniendo en cuenta las características del estudio y los antecedentes en estudios semejantes adelantados sobre Panel de Datos, la unidad de análisis son los investigadores, la unidad de medidas para las variables se calcula sobre la producción de artículos de los investigadores anualmente y la medida de las variables se fija en periodos de tres años (McFadyen y Cannella, 2005). 


\section{Tabla 1}

Distribución de la Muestra vs. Población Acotada (M/PA)

\begin{tabular}{|c|c|c|c|c|}
\hline Disciplina Región & Anglosajona & Europa & Latinoamérica & Total \\
\hline Finanzas & $50 / 200$ & $53 / 212$ & $16 / 46$ & $119 / 458$ \\
\hline Management & $52 / 208$ & 49/196 & $20 / 74$ & $121 / 478$ \\
\hline Total & $102 / 408$ & $102 / 408$ & $36 / 120$ & $240 / 936$ \\
\hline
\end{tabular}

Nota: $n=240$

Fuente: elaboración propia.

\subsection{Modelos utilizados}

Siguiendo a Martínez (2012), el estudio utilizó la técnica de panel data con modelos de efectos aleatorios construidos a partir de las ecuaciones 1, 2 y 3.

$$
\begin{aligned}
& W_{i t}=\alpha+\beta_{1} X_{1 i t}+\beta_{2} X_{2 i t}+e_{i t} \\
& Y_{i t}=\alpha+\beta_{1} X_{1 i t}+\beta_{2} X_{2 i t}+e_{i t} \\
& Z_{i t}=\alpha+\beta_{1} X_{1 i t}+\beta_{2} X_{2 i t}+e_{i t}
\end{aligned}
$$

Donde $i$ significa la $i$-ésima unidad transversal (individuos) y $t$ el periodo de medición.

\section{VARIABLES DEPENDIENTES}

$W_{i t}=$ orientación a la interdisciplinariedad del investigador $i$ en el tiempo $t$

$Y_{i t}=$ orientación a la aplicación del investigador $i$ en el tiempo $t$

$Z_{i t}=$ orientación a la interacción externa del investigador $i$ en el tiempo $t$

\section{VARIABLES INDEPENDIENTES}

$X_{1}=F($ Disciplina $)$ con disciplina $=$ Management o Finanzas

$X_{2}=F($ Región) con región = Latinoamérica o Anglosajona o Europa 


\section{PARÁMETROS}

$\beta_{1}$ y $\beta_{2}$ son parámetros 1 ó 0 para la pertenencia a una región o disciplina y e el error de estimación.

\section{VARIABLES DE CONTROL}

Tener grado de doctor y años de experiencia después de la publicación del primer artículo del investigador.

\subsection{Medidas}

Las variables se miden a partir de valores y cálculos conseguidos de las publicaciones correspondientes a los autores que componen la muestra durante el periodo de estudio y de acuerdo con los siguientes criterios y escalas.

\subsubsection{ORIENTACIÓN A LA INTERDISCIPLINARIEDAD}

La orientación a la interdisciplinariedad de un investigador se mide a partir de la interdisciplinariedad del conocimiento que el investigador incorporan en sus publicaciones. Se evalúa midiendo el porcentaje de conocimiento que proviene de categorías de conocimiento (SCs) diferentes a la categoría en la cual es reportado el artículo correspondiente (Citation Outside Category), teniendo en cuenta las Web of Knowledge Subject Categories (SCs) (Porter y Chubin, 1985). Como medida de control se utilizó la expresión (1 - Especialización), considerando esta como proxy de la interdisciplinariedad. Para esto se utilizó la ecuación de especialización considerada por Porter, Cohen, Roessner y Perreault (2007) y se encontraron resultados semejantes en los valores de interdisciplinariedad para los diferentes autores.

\subsubsection{ORIENTACIÓN A LA APLICACIÓN}

Para cuantificar la orientación a la aplicación diseñamos y validamos una escala de cinco puntos para evaluar los artículos correspondientes. La más baja orientación a la aplicación se califica con 1 y corresponde a la investigación básica pura, definida como búsqueda de compresión fundamental con ninguna orientación a uso. El nivel más alto de aplicación se califica con 5 y es entendido como la investigación puramente aplicada, con la más 
baja orientación a la compresión y la más alta orientación al uso (Stokes, 1997; Hessels, Wardenaar, Boon y Ploeg, 2014).

Verificamos la confiabilidad de la escala a partir de la lectura de los artículos por parte de un grupo de expertos principal y un grupo de control. Se realizó aplicando una prueba $t$ para comparar la igualdad de Medias entre las calificaciones del grupo principal y el grupo de control, sobre una muestra de 312 artículos. Adelantado este análisis no encontramos sustento para rechazar la igualdad de Medias. En consecuencia, consideramos que el instrumento diseñado para orientar la calificación de los artículos es confiable. El total de artículos calificados corresponde a la producción de los investigadores en el periodo de estudio 2006-2011, la cual constituye un conjunto de 1.558 artículos.

\subsubsection{ORIENTACIÓN A LA INTERACCIÓN EXTERNA}

La orientación a la interacción externa consiste en la tendencia de los investigadores a buscar coautores de lugares diferentes al lugar de trabajo habitual. Para medir esta variable se contaron los lugares de trabajo de los coautores (lugares diferentes al lugar de trabajo del autor objeto de observación) que participan en las publicaciones que hacen los investigadores (otros departamentos dentro de la misma universidad, otras universidades u otro tipo organizaciones). Después calculamos el número de lugares diferentes que se registran en las publicaciones del autor objeto de observación en un año determinado y luego determinamos el total de lugares registrados en las relaciones del autor en periodos de tres años.

\subsubsection{REGIÓN}

Esta variable corresponde a la región en la cual considera la base de datos ISI las publicaciones de los investigadores (anglosajona, Europa o Latinoamérica). Se maneja como una variable categórica y se captura involucrando en la selección de la muestra la distinción de las tres categorías en los individuos seleccionados.

\subsubsection{DISCIPLINA}

Permite distinguir la disciplina en la cual trabajan los investigadores y se categoriza para los propósitos del estudio en Management o en Finanzas. Se captura direccionando en la selección de la muestra la distinción de las dos disciplinas sobre la base de datos 
ISI y se maneja en las pruebas de hipótesis como una variable dummy para diferenciar Latinoamérica de las otras dos regiones.

\section{Resultados}

La observación final de periodo 2006-2011 muestra que en el 93,75\% de los casos la variable orientación a la interdisciplinariedad presenta una varianza menor en cada individuo que entre los individuos al final del periodo, la orientación a la aplicación en el 90,41\% de los casos y la orientación a la interacción externa en el 97,92\% de los casos. Se comprueba entonces que la varianza en el tiempo para todas las orientaciones estratégicas es mayor entre los individuos que para un mismo individuo en el periodo de estudio; se verifica así la presencia de las orientaciones estratégicas sustentadas por Martínez (2012).

\subsection{Resultados de las pruebas de hipótesis}

Hipótesis 1. Las hipótesis se prueban utilizando dos modelos para verificar en un primer modelo la influencia de las variables de control. Los resultados de los cálculos de regresión para la hipótesis 1 se presentan en la tabla 2. Teniendo en cuenta que la variable disciplina es una variable dummy, la relación negativa entre finanzas y la interdisciplinariedad señala que los investigadores en Management están más orientados a la interdisciplinariedad que los investigadores en Finanzas ${ }^{1}$. En consecuencia se sustenta la hipótesis 1: los investigadores en Management están más orientados a la interdisciplinariedad que los investigadores en Finanzas.

\section{Tabla 2}

Relación entre la disciplina de investigación y la orientación a la interdisciplinariedad (Hipótesis 1)

\begin{tabular}{lcc}
\hline Variables independientes & Mod 1-H1 & Mod 2-H1 \\
\hline \multirow{2}{*}{ Constante } & 0,5518624 & 0,592788 \\
& {$[0,034]^{* *}$} & {$[0,000]^{* * * *}$} \\
Experiencia & 0,0065459 & \\
& {$[0,000]^{* * * *}$} & \\
& & Continúa
\end{tabular}

1 Un valor negativo en el coeficiente de una variable categórica dummy es indicativo de una relación positiva entre la categoría no incluida en el modelo y la variable dependiente. 


\begin{tabular}{lcc}
\hline \multicolumn{1}{c}{ Variables independientes } & Mod 1-H1 & Mod 2-H1 \\
\hline Poseer grado de doctor & $-0,1598832$ & \\
& {$[0,542]$} & $-0,2341363$ \\
Finanzas & & {$[0,000]^{* * * *}$} \\
Wald chi2 (2) & 14,13 & 66,55 \\
Prob > chi2 & 0,0009 & 0,0000 \\
R-sq Overall & 0,0312 & 0,1606 \\
\hline
\end{tabular}

${ }^{*} \mathrm{p}<0,1 ;{ }^{* *} \mathrm{p}<0,05 ; * * * \mathrm{k}<0,01 ; * * * * \mathrm{p}<0,001$

Fuente: elaboración propia.

Hipótesis 2. Los resultados de los cálculos de regresión para la hipótesis 2 se presentan en la tabla 3. De acuerdo con los resultados se sustenta la hipótesis 2: los investigadores en Finanzas están más orientados a la aplicación que los investigadores en Management.

\section{Tabla 3}

Relación entre la disciplina de investigación y la orientación a la aplicación

(Hipótesis 2)

\begin{tabular}{lcc}
\hline Variables independientes & Mod 1-H2 & Mod 2-H2 \\
\hline Constante & 3,335581 & 3,069353 \\
& {$[0,000]^{* * * *}$} & {$[0,000]^{* * * *}$} \\
Experiencia & $-0,0039134$ & \\
Poseer grado de doctor & {$[0,435]$} & \\
& $-0,3197431$ & \\
Management & {$[0,502]$} & $-0,2010664$ \\
Wald chi2 $(2)$ & & {$[0,025]^{* *}$} \\
Prob $>$ chi2 & 1,19 & 5,03 \\
R-sq 0verall & 0,5506 & 0,0249 \\
\hline
\end{tabular}

${ }^{*} \mathrm{p}<0,1 ;{ }^{* *} \mathrm{p}<0,05 ;{ }^{* * *} \mathrm{p}<0,01 ; * * *{ }^{*} \mathrm{p}<0,001$

Fuente: elaboración propia.

Hipótesis 3. Los resultados de los cálculos de regresión para la hipótesis 3 se presentan en la tabla 4. Hay evidencia empírica para sustentar la hipótesis: los investigadores en Finanzas y Management presentan diferencias en la orientación a la interacción exter- 
na, de forma que los investigadores en Management presentan mayor orientación a la interacción externa que los investigadores en Finanzas.

\section{Tabla 4}

Relación entre la disciplina de investigación y la orientación a la interacción externa (Hipótesis 3)

\begin{tabular}{lcc}
\hline Variables independientes & Mod 1-H3 & Mod 2-H3 \\
\hline Constante & 1,5954 & 1,686473 \\
& {$[0,014]^{* *}$} & {$[0,000]^{* * * *}$} \\
Experiencia & 0,0163247 & 0,0155514 \\
& {$[0,007]^{* * *}$} & {$[0,008]^{* * *}$} \\
Poseer grado de doctor & $-0,1012921$ & \\
Finanzas & {$[0,875]$} & \\
Wald chi2 $(2)$ & & $-0,3269798$ \\
Prob $>$ chi2 & 7,39 & {$[0,006]^{* * *}$} \\
R-sq Overall & 0,0249 & 15,59 \\
\hline
\end{tabular}

${ }^{*} p<0,1 ;{ }^{* *} p<0,05 ; * * * p<0,01 ; * * * * p<0,001$

Fuente: elaboración propia.

Hipótesis 4. Los resultados de los cálculos de regresión para la hipótesis 4 se presentan en la tabla 5. La hipótesis 4 se sustenta parcialmente. Los resultados muestran que los investigadores latinoamericanos están significativamente más orientados a la aplicación que los investigadores anglosajones, pero no se puede sustentar lo mismo con relación a los investigadores europeos.

\section{Tabla 5}

Relación entre la región donde trabajan los investigadores y la orientación a la aplicación (Hipótesis 4)

\begin{tabular}{lcc}
\hline Variables independientes & Mod 1-H4 & Mod 2-H4 \\
\hline \multirow{2}{*}{ Constante } & 3,335581 & 2,936123 \\
& {$[0,000]^{* * * *}$} & {$[0,000]^{* * * *}$} \\
Experiencia & $-0,0039134$ & \\
& {$[0,435]$} &
\end{tabular}




\begin{tabular}{lcc}
\hline Variables independientes & Mod 1-H4 & Mod 2- H4 \\
\hline Poseer grado de doctor & $-0,3197431$ & \\
& {$[0,502]$} & $-0,2399118$ \\
Región anglosajona & & {$[0,097]^{*}$} \\
& & 0,0933375 \\
Región Europa & & {$[0,469]$} \\
Wald chi2 (2) & 1,19 & 6,94 \\
Prob > chi2 & 0,5506 & 0,0311 \\
R-sq Overall & 0,0011 & 0,0511 \\
\hline
\end{tabular}

${ }^{*} \mathrm{p}<0,1 ;{ }^{* *} \mathrm{p}<0,05 ; *{ }^{* *} \mathrm{p}<0,01 ; * * * * \mathrm{p}<0,001$

Fuente: elaboración propia.

\section{Discusión}

Las estadísticas descriptivas y las pruebas de las hipótesis llegan a resultados consistentes que permiten reconocer la persistencia en el tiempo de las orientaciones estratégicas de los investigadores, así como también las diferencias entre ellos en la orientación a la interdisciplinariedad, la orientación a la aplicación y la orientación a la interacción externa. En todas las pruebas se encontró que son mayores las varianzas entre los individuos que al interior de los individuos en el tiempo, verificándose con ello el sentido estratégico de las orientaciones de los investigadores.

Los resultados confirman que los investigadores en Management están más orientados a la interdisciplinariedad y a la interacción externa que los investigadores en Finanzas, quienes a su vez mostraron estar más orientados a la aplicación. De esta manera se refleja que las características particulares de las disciplinas, tales como su demarcación teórica y la incertidumbre de la tarea, influyen en las orientaciones estratégicas de los investigadores y en consecuencia en la forma de producción de conocimiento. Esto incide en que se privilegien unos rasgos de los Modos de Producción de Conocimiento sobre otros, y se tengan diferentes puntos de vista sobre el rigor y relevancia de la investigación. De hecho, en el ámbito académico es reconocido que los investigadores en Finanzas consideran poco relevante y rigurosa la investigación que utilice los métodos cualitativos que se salen de las corrientes principales de su campo de estudios y que, por el contrario, suelen ser valorados positivamente por los investigadores en Management. 
Por otro lado, tal como se esperaba, se encontró que los investigadores latinoamericanos en el CA están más orientados a la aplicación que los investigadores anglosajones, confirmando que la región incide en la escogencia que hacen los investigadores de los rasgos correspondientes a los Modos de Producción de Conocimiento. Sin embargo, no encontramos una diferencia significativa entre los investigadores latinoamericanos y los europeos en lo relacionado con la orientación a la aplicación. Es posible que esto se deba a limitaciones en el desarrollo del Management en las Escuelas de Negocios en el centro de Europa, o a políticas de investigación que han estado en muchos casos dirigidas a la investigación acción, la gestión pública y otros enfoque más orientados a la solución de problemas del estado de bienestar.

A pesar de la falta de significancia para confirmar la diferencia entre los investigadores de la Región Latinoamericana y la Región Europea, es claro que las regiones contempladas se diferencian en los modos de hacer investigación, la relevancia que sus gobiernos otorgan a la producción de conocimiento en el CA y en los recursos que otorgan para la investigación. En este sentido, los países desarrollados (anglosajones y europeos) tienden a otorgar más importancia al proceso investigativo para el desarrollo de la ciencia en sí misma que los que están en vía de desarrollo (latinoamericanos) y, por consiguiente, destinan más recursos para tal fin. La evidencia nos permite argumentar que los investigadores latinoamericanos tienen más orientación a la aplicación, debido a que sus resultados pueden ser utilizados de una manera más rápida y enfocada a resolver problemas específicos relevantes para la sociedad; en función del apoyo y los intereses gubernamentales de turno. Los investigadores anglosajones en particular tienden a ser más reflexivos y a llevar agendas con visión de largo plazo para el desarrollo teórico de la disciplina.

Sobre estos resultados sería de esperarse que los investigadores latinoamericanos produjeran conocimiento más relevante, asociado a una mayor orientación a la aplicación. No obstante, las políticas gubernamentales (ej. las de Colombia) engendran diversos problemas que hacen poner en duda no solo la relevancia sino el rigor de la investigación. Uno de ellos es justamente la poca consideración por parte de las agencias públicas de las diferencias en los Modos de Producción de Conocimiento entre disciplinas, y la falta de una evaluación rigurosa de las necesidades y prioridades en función de dichas diferencias, de forma que permitan definir los recursos apropiados para promover y evaluar de manera objetiva el desarrollo de los diferentes campos científicos. Esta circunstancia nos permite empezar a entender, en parte, por qué, paradójicamente, la mayor orientación a la aplicación no se traduce necesariamente en mayor relevancia. 
De otra parte, podemos ampliar nuestra compresión del caso latinoamericano si examinamos el problema del rigor. Nuestras observaciones mostraron que la participación comparativa de la producción de los investigadores latinoamericanos en las publicaciones de alto impacto en el CA es inferior al $15 \%$ de la producida en las tres regiones en el periodo objeto de estudio. Si se tiene en cuenta que la población de investigadores certificados por la agencia gubernamental de la ciencia en un solo país como Colombia fue de 8.000 en el año 2014, se puede sospechar que una gran parte de la investigación que se produce (o al menos la que se considera como tal) es poco rigurosa. En esta circunstancia, el dilema sobre el rigor y la relevancia en el caso latinoamericano carece de sentido pues, en el dominio de la ciencia, no puede haber relevancia sin rigor.

\subsection{Implicaciones para la teoría}

Nuestro estudio muestra que hay antecedentes de los investigadores que influyen en sus orientaciones estratégicas, lo cual a su vez incide en la aplicabilidad y el rigor de su investigación. Se estudiaron prioritariamente las influencias de la disciplina y la región donde han trabajado los investigadores y también se incluyeron la experiencia y la posesión de título de Ph.D como variables de control. La inclusión de estas últimas en algunos modelos permitió observar su influencia en las relaciones estudiadas. Por ejemplo, se encontró que los investigadores con mayor experiencia, y los pocos investigadores que no tiene título de Ph.D, están más orientados a la aplicación. Teniendo en cuenta el horizonte de tiempo que cubre la muestra y su tamaño es muy posible que las relaciones soportadas sean indicativas de una influencia causal.

La menor orientación de los investigadores en Finanzas a la interdisciplinariedad, sustentada en la primera hipótesis, pone en evidencia las consideraciones teóricas sobre el carácter más cerrado de la disciplina de Finanzas en comparación con la disciplina de Management. Los resultados de la segunda hipótesis podrían estar confirmando la devoción del Management por la teoría (Hambrick, 2007) y, consecuentemente, la más baja orientación hacia la aplicación que presentan sus investigadores, en comparación con los investigadores en Finanzas. No obstante, consideramos que para sostener esta afirmación de manera más contundente será necesario identificar y aislar en un futuro los diferentes factores que hacen que los investigadores en Finanzas se orienten más a la aplicación; tales como las demandas que hacen las empresa y los gobiernos de investigaciones sobre el comportamiento de los negocios y sus resultados en el ámbito financiero. Nuestros hallazgos contradicen en parte algunos de los discursos conocidos en la sociología de la ciencia cuando consideran que la investigación interdisciplinaria 
es la que más se orienta a la aplicación (Van den Besselaar y Heimeriks, 2001; Gibbons, 1999). Esto que parece responder al sentido común (se supone que los campos interdisciplinarios están en constante flujo para responder a los problemas sociales) resulta contradictorio cuando se observa nuestra comparación entre Management y Finanzas. Como muestran los resultados, los investigadores en Management están más orientados a la interdisciplinariedad que los investigadores en finanzas, pero no están necesariamente más orientados a la aplicación.

Observadas en conjunto, las hipótesis sustentadas muestran que las orientaciones de los investigadores constituyen dimensiones estratégicas que pueden llevar a su vez a diferentes posiciones estratégicas, dependiendo de las combinaciones que tengan en sus orientaciones. Se han validado así las tres dimensiones reconocidas en Martínez 2012 (orientación a la interdisciplinariedad, orientación a la aplicación y orientación a la interacción externa) y esto deja abierta la posibilidad de identificar las posiciones estratégicas propuestas por Kurek et al. (2007), tales como los Modos 0, 1, 2, 3, recurriendo a la información primaria que se encuentra en la base de datos ISI. Esto significa un avance metodológico importante sobre el Modelo del Posicionamiento Estratégico. De esta manera, se contribuye a fortalecer la investigación en el CA, atendiendo el rigor que le permita dialogar con investigadores posicionados en las diferentes disciplinas alrededor del mundo, y con diferentes enfoques en los Modos de Producir Conocimiento.

\subsection{Limitaciones y perspectivas para futuras investigaciones}

El análisis estadístico del presente estudio está restringido por la información que puede extraerse de la base de datos ISI. Establecimos como única fuente esta BD, dada la mayor homogeneidad de los artículos fuente y su fortaleza científica, pues se considera que las categorías ISI son unidades informativas lo suficientemente explícitas y consistentes para poder ser utilizadas en la representación de las distintas disciplinas que componen la ciencia (De Moya Anegón et al., 2006). No obstante, somos conscientes que esta condición limita el tamaño de la población de investigadores y el tamaño de la muestra. Un análisis exhaustivo por autores más allá de la producción registrada en ISI permitiría ampliar el tamaño de la población, exigir un mayor tamaño de la muestra, o incluso aumentar el volumen de datos posibles tomando la misma muestra. Este trabajo tendría que tener en cuenta los libros y otro tipo de reportes de investigación, y presentaría un conjunto de problemas que escapan al alcance de esta investigación. Otra limitación es la relacionada con el desequilibrio en las disciplinas comparadas, teniendo en cuenta que la categoría Management incluye un conjunto diverso de subcampos agrupados bajo este nombre en 
la respectiva categoría ISI (teoría y comportamiento organizacional, emprendimiento y mercadeo, entre otros). No obstante, para el estudio de las diferencias entre disciplinas abiertas y cerradas el contraste entre Management y Finanzas resulta consistente y suficiente, y sigue una tradición avanzada sobre el estudio de las disciplinas que contribuyen al CA (Whitley, 2000/1984; Vieira y Teixeira, 2010).

Otra limitación es la amplitud de las regiones consideradas. Aunque el estudio muestra evidencia sobre el efecto de lo regional en las orientaciones estratégicas de los investigadores, una distinción por países podría ofrecer resultados más precisos y conclusiones más ajustadas a cada contexto.

En general, las perspectivas para futuras investigaciones pueden orientarse a la superación de las limitaciones encontradas aquí, y pueden clasificarse en dos grandes categorías: una perspectiva de agendas generales para las tradiciones fundamentales consideradas en este estudio (p. ej., orientaciones estratégicas y modos de producción de conocimiento en administración) y una categoría de proyectos específicos que se pueden desarrollar en el corto plazo extrapolando la investigación a otras disciplinas y campos de conocimiento.

\subsection{Implicaciones para la práctica de la administración}

Los resultados de esta investigación ofrecen elementos importantes para la reflexión sobre las políticas de promoción y desarrollo de la investigación en general, y de manera particular en el CA. Consideramos que los discursos tradicionales que promueven el desarrollo del Modo 2, otros modelos prescriptivos para la creación de conocimiento (p. ej., Triple hélice y Sistemas de innovación) y los debates sobre el problema del rigor y relevancia podrían mejorar sus reflexiones introduciendo los constructos que hemos soportado en esta investigación y, en consecuencia, las prácticas que de ellos se derivan. En el caso latinoamericano, en particular, sería conveniente promover más la investigación aplicada en Management, para alcanzar la relevancia práctica esperada por la comunidad empresarial y la sociedad en general, pero sin perder de vista la necesidad de mejorar el rigor y el desarrollo conceptual del CA. En otras palabras, buscar un equilibrio entre el Modo 1 y el Modo 2 aceptando la combinación de sus atributos y dependiendo de las características y necesidades particulares de las disciplinas que contribuyen al CA.

Consideramos que las políticas específicas para promoción de grupos de investigación y la evaluación de trabajadores del conocimiento se pueden ver beneficiadas con el análisis de los resultados obtenidos en este estudio. Hemos desarrollado un método y un 
conjunto de instrumentos para determinar tres tipos de orientación estratégica de los investigadores. El método y los instrumentos desarrollados aquí son replicables en otros campos de conocimiento y pueden ayudar en el empeño de conocer más a fondo cómo se comportan los investigadores y los campos en los cuales hacen investigación.

\section{Conclusiones}

Este artículo se orientó a sustentar un modelo analítico nuevo y útil para comprobar las preferencias de los investigadores en administración por determinados rasgos de los Modos de Producción de Conocimiento. Se comprobó la presencia mixta en el nivel individual de los rasgos que definen los Modos de Producción de Conocimiento en el CA, su configuración como orientaciones estratégicas y su relación con la disciplina y la región donde ellos han trabajado de manera predominante.

Los resultados muestran que los investigadores en Finanzas y Management presentan diferencias significativas en la orientación a la interdisciplinariedad y la orientación a la aplicación, de forma que los investigadores en Management están más orientados a la interdisciplinariedad que los investigadores en Finanzas, y los investigadores en Finanzas tienen mayor orientación a la aplicación que los investigadores en Management.

También se comprobó que los investigadores presentan diferencias significativas en la orientación a la interacción externa, dependiendo de la disciplina en la cual investigan, de tal forma que los investigadores en Management están más orientados a la interacción externa que los investigadores en Finanzas. Asimismo se evidenció que los investigadores latinoamericanos están significativamente más orientados a la aplicación que los investigadores anglosajones, mostrando que las características regionales afectan las preferencias de los investigadores por determinados rasgos de los modos de producción de conocimiento.

Los hallazgos permiten cuestionar la necesidad de desarrollar las condiciones ideales del Modo 2, como requisito para producir conocimiento riguroso y relevante, y la creencia generalizada de la correlación entre la investigación interdisciplinaria y la llamada investigación aplicada. En consecuencia se reconoce que existen diferencias en el tipo de conocimiento que los investigadores valoran y buscan (más o menos orientado a la aplicación y más o menos interdisciplinario) y la forma de buscarlo (al interior de una sola organización o con otras organizaciones). Es muy probable que estas diferencias no solo se presenten entre los académicos investigadores, sino también entre los académicos y 
los profesionales no investigadores. Igualmente, es presumible que las diferencias en la forma como se busca la producción de conocimiento entre los investigadores, entre las disciplinas que aportan a la administración y entre las regiones donde se produce el conocimiento, se trasladen al dominio de todos los usuarios de la investigación y los resultados de sus decisiones.

Frente al problema del rigory la relevancia de la investigación en administración, nuestro trabajo muestra la necesidad de estudiar más a fondo las diferencias en el tipo de conocimiento que se valora y considera relevante, y la forma como los investigadores lo buscan. Así, creemos que se debería estudiar más a fondo cómo se comportan las orientaciones en los investigadores y los profesionales no académicos que estudian el emprendimiento, el comportamiento organizacional o la gerencia estratégica, entre otras disciplinas, ojalá identificando las diferencias entre los países, e identificando los diferentes impactos que se tengan en cada contexto.

En nuestro concepto, solo después de conocer a fondo el comportamiento de los investigadores en el CA, y los subcampos que contribuyen a este, se podrán construir puentes, no solo entre académicos y practicantes de la administración (p. ej., profesionales en ejercicio y formuladores de política), sino también entre los académicos que trabajan en los diferentes subcampos y entre las diferentes regiones donde se produce el conocimiento. En consecuencia, identificamos la necesidad de un programa de investigación que permita entender y cuantificar mejor la naturaleza y el grado de las brechas que pueden observarse entre los diferentes actores que intervienen en los sistemas de producción de conocimientos (conceptuales y prácticas), y con ello avanzar en la construcción de los mecanismos que contribuyan a superarlas. De esta forma, tal vez, se podrá desarrollar un enfoque más matizado para entender los componentes que influyen en la producción de conocimiento en el CA como un todo, y en la compresión multidimensional del problema del rigor y la relevancia.

\section{Referencias}

Albert, M. (2003). Universities and the market economy: The differential impact on knowledge production in sociology and economics. Higher Education, 45 (2), 147-182.

Arocena, R., and J. Sutz. (2001). Changing knowledge production and Latin American universities. Research Policy, 30 (8), 1221-1234.

Bartunek, J. M. (2011). What has happened to Mode 2? British Journal of Management, 22 (3), 555-558. 
Bonaccorsi, A. (2008). Search regimes and the industrial dynamics of science. Minerva, 46 (3), 285-315.

Burgoyne, J. G., and J. Turnbull. (2006). Towards best or better practice in corporate leadership development: Issues in Mode 2 and design science research. British Journal of Management, 17 (4), 303-316.

Calderón, G.; J. Arrubla, G. Castaño, L. Gutiérrez, R. Posada, A. Ruiz, H. Serna y J. Vivares. (2010). La investigación en administración en Colombia: condiciones para la generación de conocimiento, investigadores, institucionalización y producción científica. Medellín: Ascolfa.

Cannella, A. A., and R. L. Paetzold. (1994). Pfeffer's barriers to the advance of organizational science: A rejoinder. Academy of Management Review, 19 (2), 331-341.

Cohen, J. (1997). Statistical Power Analysis for the Behavioral Sciences. New York: Academic Press. Conpes (2009). Política Nacional de Ciencia Tecnología en Innovación. Documentos Conpes 3582. Bogotá: Consejo Nacional de Política Económica y Social, DNP.

Contreras, C.; G. Edwards y A. Mizala. (2006). La producción científica de economía y administración en Chile. Un análisis comparativo. Cuadernos de Economía, 43 (128), 331-354.

Das, T. K. (2003). Managerial perceptions and essence of the managerial word: What is an interloper business executive to make of academic-researcher perceptions of mangers? British Journal of Management, 14 (1), 23-32.

De Moya, F.; Z. Vargas, E. Chinchilla, A. Corera, F. González y J. Muñoz. (2006). Visualización y análisis de la estructura científica española: ISI Web of Science. El profesional de la información, 15 (4), 258-269.

Finnerty, J. D. (1988). Financial engineering in corporate finance: An overview. Financial Management, 14-33.

Gibbons, M.; C. Limoges, H. Nowotny, S. Schwartzman, P. Scott, and M. Trow. (1994). The New Production of Knowledge: The Dynamics of Science and Research in Contemporary Societies. Thousand Oaks, CA: Sage Publications.

Gibbons, M. (1999). Science's new social contract with society. Nature. 402, C81-C84.

Hambrick, D. (1994). What if the Academy actually mattered? Academy of Management Review, 19 (1), 11-16.

Hambrick, D. (2007). The field of management devotion to theory: too much of a good thing? Academy of Management Journal, 50 (6), 1346-1352.

Hardeman, S.; K. Frenken, 0. Nomaler, and A. Ter Wal. (2015). Characterizing and comparing Innovation systems by different 'modes' of knowledge production: A proximity approach. Science and Public Policy, 42 (4), 530-548.

Hessels, L., and H. van Lente. (2008). Re-thinking new knowledge production: A literature review and a research agenda. Research Policy, 37 (4), 740-760. 
Hessels, L. K.; T. Wardenaar, W. Boon, and M. Ploeg. (2014). The role of knowledge users in public-private research programs: an evaluation challenge. Research Evaluation, 23 (2), 103-116 doi:10.1093.

Hodgkinson, G., and K. Starkey. (2011). Not simply returning to the same answer over and over again: Reframing relevance. British Journal of Management, 22 (3), 355-369.

Huff, A. S. (2000). Changes in organizational Knowledge Production. Academy of Management Review, 25 (2), 288-293.

Huff, A. S., and J. Huff. (2001). Re- Focusing the Business School Agenda. British Journal of Management, SI, S49- S54.

Jansen, D.; R. von Görtz, and R. Heidler. (2008). Structural Holes and Knowledge Production. $5^{\text {th }}$ conference on Applications of Social Network Analysis.

Jansen, D.; von Görtz R., and R. Heidler. (2010a). Knowledge production and the structure of collaboration networks in two scientific fields. Scientometrics, 83 (1): 219-241.

Jansen, D.; von Görtz R., and R. Heidler. (2010b). Is nanoscience a Mode 2 Field? Disciplinary differences in modes of knowledge production. Chapter 3. In Governance and performance in the german public research sector. Higher Education Dynamics, 32 (2), 45-71.

Kurek, K.; P. Geurts, and H. E. Roosendaal. (2007). The research entrepreneur: Strategic positioning of the researcher in his societal environment. Science and Public Policy, 34 (7), 501-513.

Llerena, P., and F. Meyer-Krahmer. (2003). Interdisciplinary research and the organization of the university: General challenges and a case study. Science and Innovation. Recuperado de http://econpapers.repec.org/bookchap/elgeebook/2831.htm

MacLean, D.; R. MacIntosh, and S. Grant. (2002). Mode 2 Management Research. British Journal of Management, 13 (3), 189-207.

Malaver, F. (2006). El despegue de la investigación colombiana en administración: análisis de sus avances en el periodo 2000-2006. Cuadernos de Administración, 19 (32), 71-110.

Martínez, M. (2012). Creación de conocimiento en el campo de la administración: un estudio sobre la influencia de las orientaciones estratégicas de los investigadores y las redes sociales. Tesis Doctoral - Ph.D. Universidad de los Andes. Facultad de Administración - Acreditada AACSB. Bogotá, Colombia.

Martínez, M., and E. Wills. (2010 working paper). Modes of Knowledge Production, Social Networks and Knowledge Creation in Management Schools. Los Andes University. Accredited AACSB. Working Paper.

McFadyen, M. A., and A. A. Cannella. (2005). Knowledge creation and location of university research scientist' interpersonal exchange relations: Within and beyond the University. Strategic Organizations, 3 (2), 131-135.

Metzger, N., and R. Zare (1999). Interdisciplinary research: From belief to reality. Science, 283 (5402), 642-643. 
Monroy, S (2006, jul.-dic.). Nuevas políticas y estrategias de articulación del sistema de ciencia, tecnología e innovación colombiano. INNOVAR, Revista de Ciencias Administrativas y Sociales de la Universidad Nacional de Colombia, $16 \mathrm{Ed}$ (28).

Naranjo, C. y G. Calderón. (2010). La investigación en innovación en Colombia y México. Un análisis de la diseminación en Revistas Científicas. Revista Dyna, 77 (162), 191-203.

Nicolai, A., and D. Seidl. (2010). That's relevant! Different forms of practical relevance in management science. Organization Studies, 31 (9-10), 1257-1285.

Pascal, A.; C. Thomas, and A. Romme. (2013). Developing a human-centred and science-based approach to design: The knowledge management platform project. British Journal of Management, 24 (2), 264-280.

Pettigrew, A. (1997). The double hurdles for management for management research. In: T. Clarke (ed.), Advancement in Organizational Behavior (pp. 277-296). Essays in Honor of Derek S. Pugh. London: Dartmouth Press.

Pfeffer, J. (1993). Barriers to the advance of organizational science: Paradigm development as a dependent variable. Academy of Management Review, 18 (4), 599-620.

Porter, A., and D. Chubin. (1985). An indicator of cross-disciplinary research. Scientometrics, $8(3-4), 161-176$.

Porter, A.; A. Cohen, J. Roessner, and M. Perreault. (2007). Measuring research interdisciplinarity. Scientometrics, 72 (1), 117-147.

Qin, J.; F. Lancaster, and B. Allen. (1997). Types and Levels of Collaboration in Interdisciplinary Research in the Sciences. Journal of American Society for Information Science, 48 (10), 893-916.

Rousseau, D. M. (2006). Is there such a thing as "evidence-based management"? Academy of management review, 31 (2), 256-269.

Rousseau, D. M. (2007). A sticky, leveraging, and scalable strategy for high-quality connections between organizational practice and science. Academy of Management Journal, 50 (5), 1037 -1042.

Rynes, S.; T. Giluk, and K. Brown. (2007). The very separate words of academic and practitioner periodicals in human resources management: Implications for evidence - based management. Academy of Management Journal, 50 (5), 987-1008.

Simon, H. A. (1969). The sciences of the artificial. Cambridge, MA: MIT Press.

Starkey, K., and P. Madan. (2001). Bridging the relevance gap: aligning stakeholders in the future of management research. British Journal of Management, 12 (S1), S3-S26.

Stokes, D. E. (1997). Pasteur's Quadrant: Basic Science and Technological Innovation. Washington, DC: Brookings Institution Press.

Tranfield, D., and K. Starkey. (1998). The nature, social organization and promotion of management research: Towards policy. British Journal of Management, 9 (4), 341-353. 
Van Aken, J. E. (2005). Management research as a design science: Articulating the research products of mode 2 knowledge production in management. British Journal of Management, 16 (1), 19-36.

Van den Besselaar, P, and G. Heimeriks. (2001). Disciplinary, multidisciplinary, interdisciplinary. Concepts and indicators, VIII Conference on Scientometrics and Infometrics. Australia, July 16-20.

Van Leeuwen, T., and R. Tijssen. (2000). Interdisciplinary dynamics of modern science: Analysis of cross-disciplinary citation flows. Research Evaluation, 9 (3), 183-187.

Vieira, P. C., and A. C. Teixeira. (2010). Are finance, management, and marketing autonomous fields of scientific research? An analysis based on journal citations. Scientometrics, 85 (3), 627-646.

Whitley, R. (2000/1984). The intellectual and social organization of sciences, $2^{\text {nd }}$ edition, UK: 0xford University Press.

Willmott, H. (2012). Reframing relevance as 'social usefulness': A comment on Hodgkinson and Starkey's 'Not simply returning to the same answer over and over again'. British Journal of Management, 23 (4), 598-604.

Zalewska-Kurek, K. (2008). Strategies in the Production and Dissemination of Knowledge. Dissertation (PhD). Netherlands: University of Twente. 